\title{
Comparative Study between Simultaneous and Two-Stage Revascularization in Patients with Multi-Segmental Lower Limbs Arterial Occlusive Disease
}

\author{
Corresponding Author: \\ Ahmed Elsayed Afify \\ dr.ahmedsayed2013@gmail.com \\ Received for publication, March 20, \\ 2020; Accepted, July 13, 2020; \\ published online, July 13, 2020. \\ Copyright 2020 The Authors \\ published by Al-Azhar University, \\ Faculty of Medicine, Cairo, Egypt. \\ All rights reserved. This an open- \\ access article distributed under the \\ legal terms, where it is permissible \\ to download and share the work \\ provided it is properly cited. The \\ work cannot be changed in anyway \\ or used commercially. \\ doi: 10.21608/aimj.2020.26229.1174 \\ ${ }^{1}$ Vascular surgery department- \\ Faculty of medicine, Al-Azhar \\ University, Egypt.
}

Ahmed Elsayed Afify ${ }^{1}$ MSc, Mahsob Morad Amin ${ }^{1}$ MD and Mohamed Alsagheer Alhewy ${ }^{1}$ MD

\begin{abstract}
Background: Peripheral arterial disease (PAD) is defined as a slowly progressing, occlusive vascular disease of the extremities primarily due to atherosclerosis which can involve vasculitis and thrombosis and diagnosed by an ankle-brachial index (ABI) less than 0.9. It may lead to intermittent claudication (IC) and with progression of the disease may lead to critical limb ischemia (CLI).

Aim of the work: to compare the results between simultaneous revascularization of all level arterial lesions versus staged revascularization of the proximal or distal lesions regarding limb salvage rates, midterm primary patency rates, and secondary patency rates in multi-segment peripheral arterial disease

Study Design and methodology: randomized comparative study. We randomly divided patients into two groups. Group A (20 patients: treated by total revascularization of all arterial lesions affecting the target limb) and Group B (20 patients: treated staged revascularization).

Results: success rate after total revascularization (group A) reaching $82.5 \%$ success rate, success rate after staged revascularization (group B) reaching $65 \%$ success rate from inflow cases and $66.7 \%$ of the outflow repair. We achieved a limb salvage rate of $67.5 \%$ (27 patients). The difference between the groups was not statistically significant regarding amputation rates $(\mathrm{P}=0.99)$. Primary patency rate at 12 months was not significantly higher in group A than in group $\mathrm{B}(65 \%$ vs. $50 \%$; $\mathrm{P}=0.98)$. Wound closure was statistically higher in group A than in group B $(\mathrm{P}=0.04)$. Re-intervention was needed in 12 patients owing to restenosis or total occlusion of the treated lesions presenting with either lost pulses, rest pain, or deterioration of the wound healing. The secondary patency rates were statistically non-significant between group A and group B at 12 months ( 3 patients $60 \%, 2$ patients $28.6 \%)(\mathrm{P}=0.56)$. Hospital mortality was $5 \%$ in Group A and $10 \%$ in Group B. Group A showed lower amputation rate but non-significantly different with group B $(\mathrm{P}=0.5)$.

Conclusion: We conclude that multi-segmental reconstructions for multilevel occlusive arterial disease is a safe and effective method of treatment in the presence of CLI. Correction of the proximal lesions only cannot be considered satisfactory especially in patients presenting with tissue loss as it is usually associated with inferior patency rates and low overall limb salvage rates.

Keywords: Peripheral arterial disease; Angioplasty; open surgical repair; staged revascularization.
\end{abstract}

Disclosure: The authors have no financial interest to declare in relation to the content of this article. The Article Processing Charge was paid for by the authors.

Authorship: All Authors have a substantial contribution in the study

\section{INTRODUCTION}

Peripheral arterial disease (PAD) is defined as a slowly progressing, occlusive vascular disease of the extremities primarily due to atherosclerosis which can involve vasculitis and thrombosis and diagnosed by an ankle-brachial index (ABI) less than 0.9. It may lead to intermittent claudication (IC) and with progression of the disease may lead to critical limb ischemia (CLI) ${ }^{1}$. The incidence of PAD is increasing worldwide due to an overall increase in diabetes, obesity, and other cardiovascular disorders. ${ }^{2}$ Importantly, the incidence of PAD will continue to increase, as the condition has historically been diagnosed in elderly populations, which are also rising worldwide. ${ }^{3}$

Critical limb ischemia is the most advanced stage of peripheral arterial occlusive disease. The prognosis is poor, with amputation rates up to $30 \%$ and mortality up to $25 \%$ after 1 year. ${ }^{1}$

Only one out of every four to five patients with PAOD will be symptomatic. The most common 
clinical manifestation of PAOD is intermittent claudication. It is defined as ischemic pain occurring during exercise, which is quickly relieved with rest. ${ }^{4}$

\section{PATIENT AND METHODS}

Patients: This comparative study was conducted in the vascular surgery department at Al-Azhar university hospitals starting from April 2018. The study included 40 patients ( 20 patient was treated by simultaneous total revascularization \& 20 patient was treated by staged revascularizations) suffering from multisegment peripheral arterial disease. The 40 patients divided in to 2 main groups:-

Group A: This group included 20 patients with multi segmental arterial disease. For whom simultaneous total revascularization of all documented arterial lesions was done.

Group B: This group included also 20 patients with multi segmental arterial disease. For whom staged revascularizations was done.

Simultaneous or staged revascularization of all documented arterial lesions affecting the target limb with tissue loss and rest pain by endovascular treatment or by surgical repair.

All the patients subjected to the inclusion criteria mentioned below. Ethical committee had been obtained in all patients.

Inclusion criteria: Age over 18, Symptomatic, atherosclerotic multi-level arterial lesion, Critical lower limb ischemia (persistent rest pain of more than two weeks duration or ulcers or tissue loss or gangrene of one or more toes or ankle brachial Pressure Index (ABPI) $<0.4$, or peak systolic velocity (PSV)> $300 \mathrm{~cm}$ per sec) and Informed consent was provided.

Exclusion criteria: Acute limb ischemia, Presence of a metastatic malignancy, or other disease that limits life expectancy, Previous endovascular or surgical treatment of the affected side, Inability or unwillingness to complete with the follow up schedule, Pregnancy or breast-feeding, Severe renal failure or Aneurysm of the abdominal aorta .

Methods: After Informed written consent, patients was subjected to the following: Clinical evaluation. Duplex US and CT angiography. We randomly divided patients into two groups. Group A (20 patients: treated by total revascularization of all arterial lesions affecting the target limb) and Group B (20 patients: treated staged revascularization). In certain conditions we only able to treat the proximal lesions leaving the more distal lesions without intervention. These conditions include extended time of procedure, use of large amount of contrast or bad general condition of the patient during or after the procedure. Each group treated by angioplasty with or without stenting or by bypass surgery by using polytetrafluoroethylene grafts or saphenous graft. Follow up and assessment:

The patients was examined at the time of randomization, and after 1 month, 3 months, 6 months and 1 year. At the initial visit, medical history obtained, clinical, duplex and CT angiography was performed. Clinical improvement was documented before discharge and at subsequent outpatient visits.
Wound closure and limb salvage are our primary endpoint. Clinical improvement was judged by palpable peripheral pulse, increase of claudication distance, disappearance of rest pain, wound healing, and limb salvage. The results obtained were statistically evaluated by SPSS 20 . The study was approved by the Ethics Board of Al-Azhar University.

\section{RESULTS}

The present study was conducted on 40 patients, 22 males $(55 \%)$ and 18 females (45\%). Figure 1 shows this distribution. Age of patients was $60.7 \pm 12.35$ in total revascularization group and $66.2 \pm 11.24$ in Staged revascularization ( $>00.05)$. Their age ranged between 40 years and 80 years with a mean age of $60.7 \pm 12.35$.

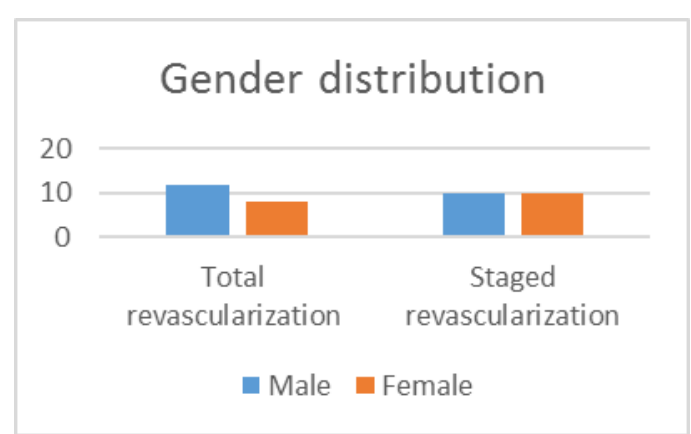

Fig. 1: Distribution of the studied patients regarding their sex.

The associated comorbidities are shown in (Table 1)

\begin{tabular}{|c|c|c|}
\hline & Total & Staged \\
\hline IHID & 4 & $\mathbf{8}$ \\
\hline C.V.S. & 2 & 2 \\
\hline D.M. & 10 & $\mathbf{1 5}$ \\
\hline Smoking & 9 & $\mathbf{1 0}$ \\
\hline Hypertension & 8 & $\mathbf{1 1}$ \\
\hline Dyslipidemia & 12 & $\mathbf{1 1}$ \\
\hline p-value & \multicolumn{2}{|c|}{$\mathbf{0 . 0 5}$} \\
\hline
\end{tabular}

Table 1: The associated diseases $\&$ risk factors in the patients

Symptoms and signs of patients included in this study included Rest pain, Ischemic Ulcers, Gangrene of Toe, Heel or Forefoot as shown in (Table 2).

\begin{tabular}{|c|c|c|}
\hline Symptoms \& signs & Total & Staged \\
\hline Rest pain & 15 & 12 \\
\hline Ischemic Ulcers & 6 & 5 \\
\hline Gangrene: & 14 & 15 \\
Toe & 8 & 10 \\
Heel & 1 & 3 \\
Forefoot & 5 & 2 \\
\hline
\end{tabular}

Table 2: Symptoms and signs of patients included in this study

II-Lesion characterizations' in (Tables 3,4)

A total number of 56 multi-segmental reconstructions were performed for 40 patients (sixteen had bilateral 
procedures) for treatment of critical limb ischemia due to multi segmental occlusive arterial disease .

In 20 cases, revascularization was performed in one stage (Group A) and in 20 cases - as a two-stage procedure (Group B)

Ante-grade approach was used in 17 (70.8\%) patients, divided into ipsilateral femoral access in 10 patients

Group A: (Total revascularization)

\begin{tabular}{|c|c|}
\hline Lesion \& Operation & Number of cases \\
\hline \multirow{3}{*}{$\begin{array}{l}\text { Aortoiliac + SFA } \\
\text { - Aorto-bifem. +Fem-pop. } \\
\text { - Kissing stent+Fem-pop. }\end{array}$} & 8 \\
\hline & 5 \\
\hline & 3 \\
\hline SFA + Tibial & 8 \\
\hline \multirow{2}{*}{$\begin{array}{l}\bullet \quad \text { Fem-pop. } \\
\text { Supragenicular+Tibial } \\
\text { Angioplasty }\end{array}$} & 3 \\
\hline & 5 \\
\hline - $\quad$ SFA + Tibial Angioplasty & \\
\hline \multirow{3}{*}{$\begin{array}{c}\text { Iliac }+ \text { Common Femoral } \\
\bullet \text { Ilio-Femoral Bybass } \\
\bullet \quad \text { Angioplasty }\end{array}$} & 4 \\
\hline & 3 \\
\hline & 1 \\
\hline
\end{tabular}

Table 3: Lesion characterization and interventions of group A

Group B: (Staged revascularization)

\begin{tabular}{|l|l|}
\hline \multicolumn{2}{|c}{ Aortoiliac + SFA (8 cases) } \\
\hline Stage I (8 cases) & Stage II (2 cases) \\
\hline Aorto-bifem. (6 cases) & Fem-pop (1 case) \\
\hline Kissing stent ( 2 cases) & Fem-pop (1 case) \\
\hline SFA + Tibial (6 cases) & Stage II (5 cases) \\
\hline Stage I (6 cases) & Tibial Angioplasty ( 2 cases) \\
\hline Fem-pop. Supragenicular (3 cases) & Tibial Angioplasty (3 cases) \\
\hline SFA Angioplasty (3 cases) & \\
\hline Iliac + SFA (6 cases) & Stage II (1 cases) \\
\hline Stage I (6 cases) & Fem-pop (1 case) \\
\hline Ilio-Femoral Bypass. ( 4 cases) & Fem-pop (0 case) \\
\hline Iliac Angioplasty (2 cases) & \\
\hline Table 4: Lesion characterization and interventions of group B & \\
\hline
\end{tabular}


Primary patency rate at 12 months was not significantly higher in group A than in group B (65\% vs. $50 \%$; $\mathrm{P}=0.98)$

\begin{tabular}{|l|l|l|l|}
\hline Mean procedure time & $\begin{array}{l}\text { Total } \\
\text { revascularization }\end{array}$ & $\begin{array}{c}\text { Staged } \\
\text { revascularization } \\
\text { Stage I }\end{array}$ & Stage II \\
\hline Aortoiliac + SFA & $3.2 \pm 1.8$ & $2.9 \pm 1.3$ & $1.9 \pm 1.1$ \\
\hline SFA + Tibial & $2.6 \pm 1.5$ & $1.8 \pm 1.1$ & $1.0 \pm 0.6$ \\
\hline Iliac +fem. & $2.6 \pm 1.8$ & $2.1 \pm 1.0$ & $2.0 \pm 1.2$ \\
\hline
\end{tabular}

Table 5: Procedure time (hours)

Procedure (Technical) outcome:

Success rate after total revascularization (group A) was $82.5 \%$ and $65 \%$ after staged revascularization (group B) from inflow cases and $66.7 \%$ of the outflow repair.

Mean operation time was calculated for each procedure and exact numbers are shown in (Table 5)

Mean hospital stay was calculated for each procedure and exact numbers are shown in Antegrade approach was used in $17(70.8 \%)$ patients, divided into ipsilateral femoral access in 10 patients and brachial access in 7 patients (using long sheath), whereas contralateral access with cross-over sheath was used in 7 (29.2) patients. The lesions were crossed intraluminal in 10 (41.7) patients and sub-intimal in 14 (58.3) patients. Stents were used in $12(50 \%)$ patients.

\section{Clinical outcome:}

We achieved a limb salvage rate of $67.5 \%$ (27 patients). With 15 patients $(75 \%)$ in group A and 12 patients $(60 \%)$ for group B and a major amputation rate of $32.5 \%$ (13 patients) over 1-year follow-up period (Figure 2).

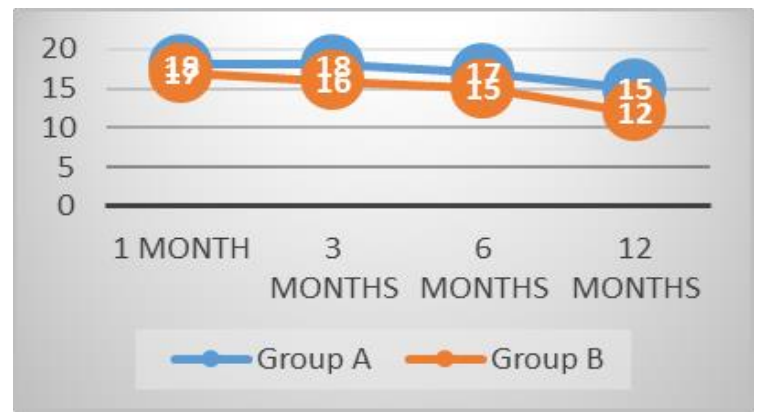

Fig. 2: Limb salvage rate over one year period

There were two limbs lost in the early postoperative period in Group A (10\%) due to thrombosis of the grafts and advanced gangrene of the foot. In Group B, three limbs were lost during the first month after operation (15\%).

In the remote postoperative period, limb salvage was $75 \%$ after 12 months in Group A and 60\% in Group B.

During the observation period, a total number of 5 limbs were lost (25\%) in Group A and 8 (40\%) in Group B. The difference between the groups was not statistically significant $(\mathrm{P}=0.99)$. (Figure 3 )

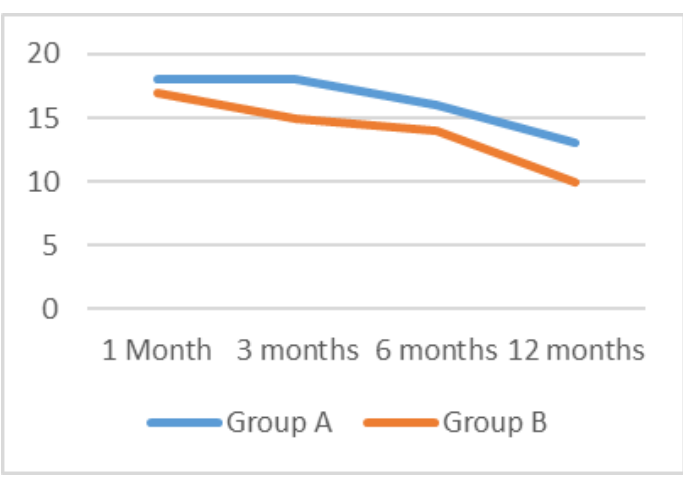

Fig. 3: Primary patency rate

The primary graft patency rate was $65 \%$ in Group A and $50 \%$ in Group B after one year. The difference between the groups was not statistically significant $(\mathrm{P}=0.98)$.

Wound closure was statistically higher in group A than in group $\mathrm{B}(\mathrm{P}=0.04)$. (Figure 4$)$

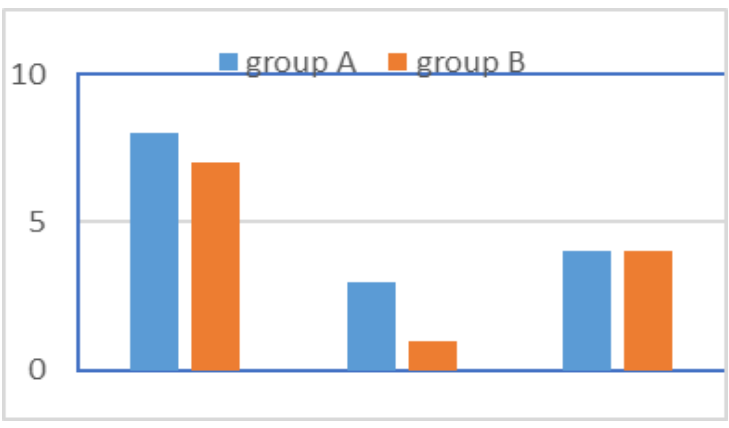

Fig. 4: Wound closure Comparison

\section{2ry patency at 1 year:}

Re-intervention was needed in 12 patients owing to restenosis or total occlusion of the treated lesions presenting with either lost pulses, rest pain, or deterioration of the wound healing. The secondary patency rates were non statistically significant between group and group B at 12 months ( 3 patients $60 \%, 2$ patients $28.6 \%)(\mathrm{P}=0.56)$. (Figure 5)

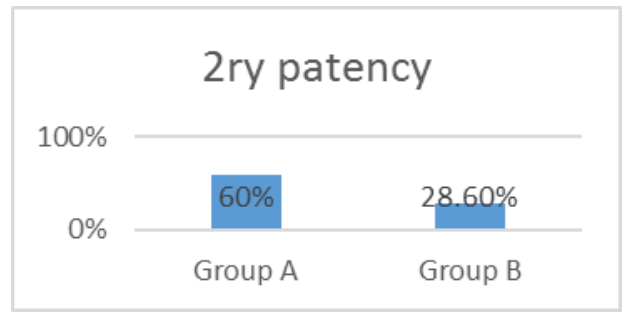

Fig. 5: 2ry patency rate 
We had registered 17 cases of graft thrombosis - 5 cases of thrombosis of both inflow and outflow grafts, 9 cases of thrombosed outflow grafts, and 3 cases of thrombosed inflow grafts.

Re-operation consisted of revision or thrombectomy of the inflow graft. All 5 cases of thrombosis of inflow and outflow grafts were operated and restoration of blood flow in both grafts was attempted.

In 9 cases, we registered isolated occlusion of the outflow grafts. In 3 cases, it was detected during routine examination and there was no evidence of critical ischemia of the limb. In these cases, surgery was not offered. In 6 cases of isolated occlusion of the outflow graft, angiography was done.

Revascularization was attempted in 12 cases and was successful in 5 cases. The secondary graft patency rate was $60 \%$ in Group A and $28.6 \%$ in Group B after one year. The difference between the groups was not statistically significant after follow-up $(\mathrm{P}=0.56)$.

Vessel perforation occurred in three patients, one in anterior tibial artery (ATA) and two in posterior tibial artery (PTA), and all were managed by prolonged balloon inflation.

Mortality

There was one early postoperative ( $<24$ hours) death in Group B due to intraoperative myocardial ischemia. Hospital mortality was 5\% in Group A (one death) and 10\% in Group B (two deaths). Causes of death were the following: two of myocardial infarction and one of bronchopneumonia.

Amputation

Minor and major limb loss were shown in (Table 6,7).

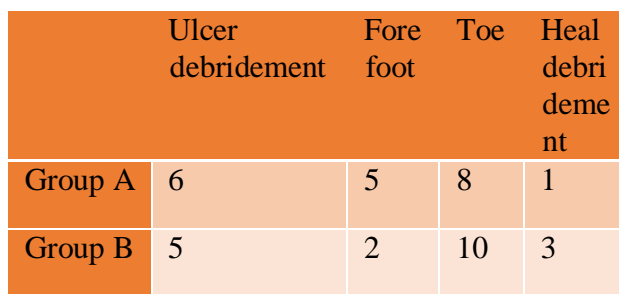

Table 6: Minor limb loss

\begin{tabular}{|l|l|l|}
\hline & AKA & BKA \\
\hline Group A & 3 & 2 \\
\hline Group B & 5 & 3 \\
\hline
\end{tabular}

Table 7: Major limb loss

Above-knee amputation was done for $8(20 \%)$ patients and below-knee amputation for $5(12.5 \%)$ patients in our study. Group A showed lower amputation rate but nonsignificantly different with group $\mathrm{B}(\mathrm{P}=0.5)$.

Practical results:-

The disappearance of ischemic rest pain and healing of gangrenous and trophic ulcers after minor (toes or transmetatarsal) amputations or debridement was considered as a positive result.

A total amount of 40 minor amputations or debridement were performed after reconstructive surgery. In all; significant improvement was achieved in patients with functioning grafts. Three diabetic patients, despite functioning grafts, lost their limbs because of widespread infection of the foot.

Postoperative improvement of $\mathrm{ABI}$ was regarded as an objective indication of improved perfusion of the limb. ABI increased from $0.29 \%$ before the operation to $0.89 \%$ in Group A and from $0.37 \%$ to $0.63 \%$ after inflow and to $0.85 \%$ after outflow procedures in Group B. The difference between the groups was statistically significant $(\mathrm{P}=0.047)$.

\section{DISCUSSION}

Surgical treatment of CLI is based on correction of the most affected segment. Identification of hemodynamically significant arterial lesions can be difficult in the presence of multilevel disease. ${ }^{1}$

We tried to select the criteria for multi-segmental reconstruction based on simple, most widely used and inexpensive clinical vascular laboratory and angiographic findings as in Langsfeld et al, Taylor et al. ${ }^{5}$

In case of critical limb ischemia, many authors as Baker et al, Flanigan et al indicate the importance of the extent of disease in the deep femoral artery which forms the main collateral network in the presence of superficial femoral artery occlusion. Occlusion of diffuse diseased PFA is one of the most important indications to perform multisegmental reconstruction. , $^{6,7}$

Diabetic vasculopathy is the most common reason of mortality and morbidity in diabetes and is responsible for high incidence of vascular diseases such as stroke, myocardial-infarction, and peripheral vascular diseases. ${ }^{8}$

Diabetics are up to 15 times more likely than non-diabetics to experience a major amputation. Diabetes is also associated with decreased primary patency following endovascular interventions.

Endovascular interventions for CLI continue to have variable reported results, adequate rates of limb salvage can be achieved in patients undergoing multilevel interventions for CLI, and improved patency is seen with multilevel compared with isolated tibial interventions. ${ }^{8}$

On the other hand, there is no doubt that healing of the foot in the presence of tissue loss and gangrene requires close to normal blood supply in the damaged area. Some authors indicate that in these conditions, postoperative $\mathrm{ABI}$ index is supposed to be raised to 0.80 or more. We believe that patients with tissue loss of the foot and the presence of multilevel occlusive disease are the most serious candidates for multi-segmental reconstructive surgery. ${ }^{8}$

Our study included 40 patients of different age and sex suffering from multi-segment peripheral arterial disease.

A total number of 56 multi-segmental reconstructions were performed for 40 patients (sixteen had bilateral procedures) for treatment of critical limb ischemia due to multi segmental occlusive arterial disease.

In 20 cases, revascularization was performed in one stage (Group A) and in 20 cases - as a two-stage procedure (Group B).

All patients enrolled in our study had associated comorbidities and risk factors (e.g. Diabetes, hypertension, ischemic heart disease, smoking, renal impairment, stroke, 
or chest disease), which were presented in the study as numbers of comorbidities and risk factors for each patient. Among our 40 patients with critical lower limb ischemia success rate after total revascularization (group A) reaching $82.5 \%$ success rate.

Success rate after staged revascularization (group B) reaching $65 \%$ success rate from inflow cases and $66.7 \%$ of the outflow repair.

We achieved a limb salvage rate of $67.5 \%$ (27 patients). With 15 patients $(75 \%)$ in group A and 12 patients $(60 \%)$ for group B and a major amputation rate of $32.5 \%$ (13 patients) over 1-year follow-up period.

During the observation period, a total number of 5 limbs were lost (25\%) in Group A and 8 (40\%) in Group B. The difference between the groups was not statistically significant

Dalman et al. presents a $90 \%$ limb salvage rate at the 36 month follow-up period in his group of 62 patients. It needs to be noted that $20 \%$ of patients in this group were operated on for claudication. ${ }^{9,10}$

Charlesworth et al. demonstrates excellent limb salvage rates in his series of 153 patients. During a five-year followup period, only seven limbs were lost. But in this group, patients were selected for aorto-femoro-popliteal reconstruction only in ischemic rest pain; patients with gangrene were not included. Not a single femoro-tibial bypass was performed in this group. ${ }^{10}$

Batt et al., in his series of 31 patients, reported five early amputations $(16.1 \%)$ and at the end of the study of 48 months, seven limbs were lost $(22.5 \%)$. Kram et al. presents a 78\% five-year limb salvage rate after unilateral aortofemoro-popliteal distal bypass. Ellenby et al. presented with a $77 \%$ limb salvage rate at five years in a group of 21 patients after femoro-popliteal bypass $.9,11$

We also found that increasing numbers of risk factors and comorbidities had drawback on the primary patency rate at 3 month and 12 month and on limb salvage rates with increase in the amputation rate.

Abularrage et al. reviewed that diabetes mellitus is an independent predictor of decreased long-term primary patency after PTA/stent in 920 patients who underwent $1075 \mathrm{PTA} /$ stent procedures, and long-term limb salvage remains inferior in diabetic patients compared with nondiabetic patients owing to a more severe clinical presentation and poor run-off.$^{12,19}$

In his study considered superficial femoral plus iliac lesions in addition to age, dialysis, left ventricular dysfunction, diabetes, hematoma prolonging hospitalization, and coronary artery disease as positive predictors of all-cause mortality.

Similar to our results, two studies of patients with multilevel lower limb arterial disease showed significantly improved secondary patency rates of multilevel intervention compared with single-level intervention.

Sadek et al. in a study for treatment of multilevel lower limb arterial lesions on 85 patients mentioned a limb salvage rate of $76.1 \%$, similar to our limb salvage rate of $75 \%$ in their group A patients showed significantly higher limb salvage rate and significant lower amputation rate than group B.
Fernandez et al. showed a limb salvage rate of $95 \%$ for multi-level arterial lesions $(\mathrm{P}=0.05) .^{21}$

Wound healing was achieved in $75 \%$ in group $A$ and $60 \%$ in group $\mathrm{B}$ with multilevel disease at 1 year. $(\mathrm{P}=0.05)$. Guo et al. in a study including 53 patients with TASC II D femoro popliteal occlusive disease showed a technical success rate of $95 \%$ with mean follow-up period of $12.2 \pm 6.1$ months (538 months). Primary patency rate at 1 year was $63 \%$, and secondary patency rate at 1year was $96 \%$. These results were significantly better than our results because they included lesions in femoro popliteal segment only which was treated mainly by primary stenting whereas other levels of arterial lesion were not included.

We also noticed that patients who presented with minor tissue loss or rest pain showed significantly higher limb salvage rates whereas patients who presented with major tissue loss or heal ulcer showed higher amputation rates. These findings were reported before in a study by Ghoneim et al.

The primary graft patency rate was $65 \%$ in Group A and $50 \%$ in Group B after one year. The difference between the groups was not statistically significant $(\mathrm{P}=0.98)$.

Graft patency rates observed by Dalman et al. were $92.6 \%$ for inflow grafts and $94.9 \%$ for outflow grafts after 24 months. Eidt et al. present a 94\% aorto-femoral graft patency rate after five years, while femoro-popliteal graft patency rate was $80 \%$ for the saphenous vein after four years and $50 \%$ for polytetrafluoroethylene (PTFE) grafts after two years .10

Batt et al ${ }^{9}$ presents a $40 \%$ bypass patency rate at four years. Using an extra-peritoneal iliac endarterectomy as an inflow procedure combined with femoro-popliteal/tibial bypass, Taylor et al. achieved $100 \%$ patency of the iliac artery and an $87 \%$ outflow patency rate at six years. After -femoropopliteal reconstruction, Ellenby et al. presented a $40 \%$ patency rate of the femoro-popliteal segment after five years. $^{11}$

The secondary graft patency rate was $60 \%$ in Group A and $28.6 \%$ in Group B after one year. The difference between the groups was not statistically significant after one year of follow-up $(\mathrm{P}=0.56)$.

Reintervention was needed in 12 patients owing to restenosis or total occlusion of the treated lesions presenting with either lost pulses, rest pain, or deterioration of the wound healing. The secondary patency rates were non statistically significant between group and group B at 12 months (3 patients $60 \%, 2$ patients $28.6 \%$ ).

We had registered 17 cases of graft thrombosis - 5 cases of thrombosis of both inflow and outflow grafts, 9 cases of thrombosed outflow grafts, and 3 cases of thrombosed inflow grafts.

Re-operation consisted of revision or thrombectomy of the inflow graft. All 5 cases of thrombosis of inflow and outflow grafts were operated and restoration of blood flow in both grafts was attempted.

In 9 cases, we registered isolated occlusion of the outflow grafts. In 3 cases, it was detected during routine examination and there was no evidence of critical ischemia of the limb. In these cases, surgery was not offered. In 6 
cases of isolated occlusion of the outflow graft, angiography was done.

Our results are favorably comparable with those of other authors as Batt $\mathrm{M}$ et al, Eidt $\mathrm{J}$ et al, O'Donnel et al. ${ }^{10,20}$

Active surgical treatment of occluded grafts is now the accepted standard for vascular surgeons. Cheshire et al., in a series of 395 patients operated on for CLI, found that failure to re-intervene after primary graft failure would reduce limb salvage at three years to $70 \%$.

We fully support authors' opinions Ellenby et al, Harrington et al, Kram et al regarding aggressive re-interventions in the presence of graft failure. In most cases, when the patient presented with ischemia of the limb due to graft thrombosis, an operation to restore the blood flow was attempted. ${ }^{11}$

Vessel perforation occurred in three patients, one in anterior tibial artery (ATA) and two in posterior tibial artery (PTA), and all were managed by prolonged balloon inflation.

There was one early postoperative ( $<24$ hours) death in Group B due to intraoperative myocardial ischemia. Hospital mortality was 5\% in Group A (one death) and 10\% in Group B (two deaths). Causes of death were the following: two of myocardial infarction and one of bronchopneumonia.

Above-knee amputation was done for 8 (20\%) patients and below-knee amputation for $5(12.5 \%)$ patients in our study. Group A showed lower amputation rate but nonsignificantly different with group $\mathrm{B}(\mathrm{P}=0.5)$.

The disappearance of ischemic rest pain and healing of gangrenous and trophic ulcers after minor (toes or transmetatarsal) amputations or debridement was considered as a positive result.

A total amount of 40 minor amputations or debridements were performed after reconstructive surgery. In all; significant improvement was achieved in patients with functioning grafts. Three diabetic patients, despite functioning grafts, lost their limbs because of widespread infection of the foot.

Postoperative improvement of $\mathrm{ABI}$ was regarded as an objective indication of improved perfusion of the limb. ABI increased from $0.29 \%$ before the operation to $0.89 \%$ in Group A and from $0.37 \%$ to $0.63 \%$ after inflow and to $0.85 \%$ after outflow procedures in Group B. The difference between the groups was statistically significant $(\mathrm{P}=0.047)$.

\section{CONCLUSIONS}

All efforts should be exerted to achieve total correction of all lesions to secure good in-line pulsatile flow to the foot. As it is associated with better primary and secondary patency rates and significantly higher limb salvage rates than correction of the proximal arterial lesions only.

Study concludes that multi-segmental reconstructions for multilevel occlusive arterial disease is a safe and effective method of treatment in the presence of CLI. Perioperative mortality, limb salvage and graft patency rates are better in the group of simultaneous reconstructions.

Correction of the proximal lesions only cannot be considered satisfactory especially in patients presenting with tissue loss as it is usually associated with inferior patency rates and low overall limb salvage rates.

\section{REFERENCES}

1. Norgren $\mathrm{L}$ and William R: Inter-society consensus for the management of peripheral arterial disease (TASC II Eur.J.Vasc.Endovasc.Surg.,2007, 43:. 71-75

2. Pattillo, C. B., Bir, S., Rajaram, V and Kevil, C. $\mathrm{G}$ Inorganic nitrite and chronic tissue ischaemia: a novel therapeutic modality for peripheral vascular diseases. Cardiovascular Research, 2011, 89: pp. 533-41. doi:10.1093/cvr/cvq297.

3. Joost A Bekken, Jan Albert Vos, Ruud A Aarts, Jean-Paul PM de Vries and Bram Fioole Department of Vascular Surgery, Rotterdam, 2012, 3079 DZ, the Netherlands.

4. Abularrage CJ, Conrad MF, Hackney LA, Paruchuri V, Crawford RS, Kwolek CJ, et al. Long-outcomes of diabetic patients undergoing endovascular infrainguinal interventions term. $J$ Vasc Surg 2010; 52:314-322.

5. Taylor LM Jr, Freimanis IE, Edwards JM, et al. Extraperitoneal iliac endarterectomy in the treatment of multilevel lower extremity arterial occlusive disease. Am J Surg 2015; 152:34-9.

6. Baker JD. Hemodynamic assessment of aortoiliac segment. Surg Clin N Am 2010; 70:3140.

7. Flanigan DP, Ryan TJ, Williams LR, et al. Aortofemoral or femoropopliteal revascularization? A prospective evaluation of the papaverine test. $J$ Vasc Surg 2006;1:215-23.

8. Flanigan DP, Williams LR, Scwartz JA, et al. Hemodynamic evaluation of the aortoiliac system based on pharmacologic vasodilation. J Vasc Surg 2012;93:709-14.

9. Batt $M$, Declemy $S$, Persch $M$, et al. Synchronous reconstruction for combined aortoiliac and femoropopliteal occlusive lesions. J Cardiovasc Surg 2010;31:448-52.

10. Eidt J, Charlesworth D. Combined aortobifemoral and femoropopliteal bypass in the management of patients with extensive atherosclerosis. Ann Vasc Surg 2008; 1:453-60.

11. Ellenby MI, Sawchuk AP, Scwarcz TH, et al. A nine year experience with crossover femorofemoropopliteal sequential bypass. Am J Surg 2011; 161:672-6.

12. Fernandez N, McEnaney R, Marone LK, Rhee RY, Leers S, Makaroun M, Chaer RA. Multilevel versus isolated endovascular tibial interventions for critical limb ischemia. J Vasc Surg 2011; 54:722-729.

13. Ghoneim B, Elwan H, Eldaly W, Khairy H, Taha A, Gad A. Management of critical lower limb ischemia in endovascular era: experience from 511 patients. Int J Angiol 2014; 23:197-206. 
14. Guo X, Shi Y, Huang X, Ye M, Xue G, Zhang J. Feature's analysis of lower extremity arterial lesions in 162 diabetes patients. J Diabetes Res 2013; 2013:781360.

15. Guo X, Xue G, Huang X, Xie H, Liang W, Zhang $\mathrm{J}$, et al. Outcomes of endovascular treatment for patients with TASC II D Femoropopliteal occlusive disease: a single center study. BMC Cardiovasc Disord 2015; 15:44-50.

16. Harrington ME, Harrington EB, Haimov M, et al. Iliofemoral versus femorofemoral bypass: the case for an individualized approach. $J$ Vasc Surg 2013; 16:841-54.

17. Kram HB, Gupta SK, Veith FJ, et al. Unilateral aortofemoral bypass: a safe and effective option for the treatment of unilateral limb-threatening ischemia. Am J Surg 2010; 162:155-8.

18. Langsfeld M, Nepute J, Hershey FB, et al. The use of duplex scanning to predict hemodynamically significant Aortoiliac stenoses. J Vasc Surg 2007; 7:395-9.

19. Miura T, Soga Y, Miyashita Y, lida O, Kawasaki D, Hirano K, et al. Five-year prognosis after endovascular therapy in claudicant patients with iliofemoral artery disease. J Endovasc Ther 2014; 21:381-388.

20. O'Donnel FT Jr, McBride KA, Callow AD, et al. Management of combined segment disease. Am J Surg 1981; 141:452-9.

21. Sadek M, Ellozy SH, Turnbull IC, Lookstein RA, Marin ML, Faries PL. Improved outcomes are associated with multilevel endovascular intervention involving the tibial vessels compared with isolated tibial intervention. $J$ Vasc Surg 2009; 49:638-643. 